[3] J. Dugundji, Topology, Boston 1970.

[4] F. T. Farrell, L. R. Taylor and J. B. Wagoner, The Whitehead theorem in the proper category, Comp. Math. 27 (1973), pp. 1-23.

[5] S. Mardešić, On the Whitehead theorem in shape theory I, Fund. Math. 91 (1976), pp. $51-64$.

[6] - On the Whitehead theorem in shape theory II, Fund. Math. 91 (1976), pp. 93-103.

[7] - and $\breve{S}$. Ungar, The relative Hurewicz theorem in shape theory, preprint.

[8] K. Morita, Cech cohomology and covering dimension for topological spaces, Fund. Math. 87 (1975), pp. 31-52.

[9] - On shapes of topological spaces, Fund. Math. 86 (1975), pp. 251-259.

[10] M. Moszyńska, The Whitehead theorem in the theory of shape, Fund. Math. 80 (1973), pp. 221-263.

[11] L. R. Taylor, Surgery on Paracompact Manifolds, thesis, University of California at Berkeley, 1971.

[12] J. H. C. Whitehead, Combinatorial homotopy I and II, Bull. Amer. Math. Soc. 55 (3) and (5) (1949)

\section{One-to-one Carathéodory representation theorem for multifunctions with uncountable values}

by

\section{A. D. Ioffe (Moscow)}

Abstract. It is shown that, given a measurable set-valued mapping $M$ from a complete measure space $T$ into a Polish space $X$ such that all sets $M(t)$ are uncountable, there are another Polish space $Z$ and a one-to-one Carathéodory mapping $f: T \times Z \rightarrow X$ such that $f(t, Z)=M(t)$ for all $t$.

8 1. Introduction. In [6] Wesley proved that, given a set-valued mapping $M$ from $I$ into $I$ ( $I$ being the unit real interval) with Borel graph and uncountable values, there into $I$ (I being the $f: I \times I \rightarrow I$ which is Lebesgue measurable in the first variable and Borel isomorphism from $I$ onto $M(t)$ in the second. Cenzer and Mauldin [1] strengthened this result having shown that, in the first argument, $f$ can be chosen measurable with respect to the minimal $\sigma$-algebra containing Borel subsets of $I$ and closed under $A$-operation of Souslin (which is a proper subalgebra of the algebra of Leunder $A$-operation of sets). On the other hand, we proved in [2] that, given a multifunction $M$ from a measurable space $(T, \mathfrak{M})$ into a Polish space $X$ with Souslin (in an appropriate sense) graph, there are another Polish space $Z$ and a Carathéodory function $f: T \times Z \rightarrow X$ such that $f(t, Z)=M(t)$ for all $t$ (such that $M(t) \neq \varnothing$ )

The question arises if and how both results can be united. For instance, is it possible to replace in Wesley's theorem "Borel isomorphism" by "one-to-one and continuous"? An affirmative answer will be given here even in a more general setting that in [6] though not in so general as in [2]. The result to be proven here is stated as follows.

THEOREM. Let $(T, \mathfrak{M}, \mu)$ be a measure space with $\sigma$-finite complete positive measure, and let $X$ be an uncountable Polish space. Let $M$ be a set-valued mapping from $T$ into $X$ such that

(i) every $M(t)$ is an uncountable subset of $X$;

(ii) $\operatorname{Gr} M=\{(t, x) \in T \times X \mid x \in M(t)\}$, the graph of $M$, belongs to $\mathfrak{M} \otimes \mathfrak{B}(X)$.

Then $M$ can be represented by a pair $(Z, f)$, where $Z$ is a Polish space, $f: T \times Z \rightarrow X$ is a Carathéodory function and for any $t \in T$, the mapping $z \rightarrow f(t, z)$ is one-to-one. 
Saying that $(Z, f)$ represents $M$, we mean that $M(t)=f(t, Z)=\{x \mid x=f(t, z)$ for some $z \in Z\}$ for any $t \in T$. A Carathéodory function is one which is measurable in the first variable and continuous in the second. By $\mathfrak{B}(X)$ we denote the $\sigma$-algebra of Borel subsets of $X$ and $\mathfrak{M} \otimes \mathfrak{B}(X)$ is the product algebra, that is, $\sigma$-algebra generated by all products $G \times B$, where $G \in \mathfrak{M}$ and $B \in \mathfrak{B}(X)$.

The theorem stated above extends Wesley's result in two directions. The most principal extension is that in our case continuous mappings $z \rightarrow f(t, z)$ appear, not Borel. The other extension is that we replace the first $I$ by an arbitrary measure space with complete $\sigma$-finite measure. The fact that the second $I$ is replaced by an arbitrary uncountable Polish space is of course of no importance (in the framework of Wesley's result) since any such space is Borel isomorphic to $I$.

However we failed to replace $(T, \mathfrak{M}, \mu)$ by a measurable space of a more general type, say closed under $A$-operation but not necessarily connected with a complete measure. (Thus our theorem does not fully covers the theorem of Cenzer and Mauldin.) The unsolved problem lying behind can be formulated as follows. Given a measurable set-valued mapping from a measurable space $(T, \mathfrak{M})$ into a Polish space, the values of the mapping being closed countable sets. Can it be exhausted by a countable family of measurable selections? As shown by Lusin [4], the answer is yes if $T$ itself is a Polish space and $\mathfrak{M}=\mathfrak{B}(T)$. This result, however, is not very much helpful since countably-valued mapping arising in situations similar to those considered here are usually measurable with respect to richer algebras. The answer is also yes in the case of complete measure space, as it follows implicitly from the proof (Propositions (n) and (o), §3). Observe that in both cases the proof is not effective.

The proof of the theorem will be given in $\S 3$. The next section contains a description of an auxiliary device.

§ 2. $\mathscr{T}$-operation. In what follows, $N=\{1,2, \ldots\}$ and $\mathscr{N}=N^{N}$ is the Baire space of countable sequences of positive integers $i=\left(i_{1}, i_{2}, \ldots\right)$. The metric

$$
b(i, j)=\sum_{k=1}^{\infty} 2^{-k} \frac{\left|\dot{i}_{k}-j_{k}\right|}{1+\left|i_{k}-j_{k}\right|}
$$

turns $\mathscr{N}$ into a complete separable metric space. It is well known that every Polish space is a one-to-one continuous image of a closed subset of $\mathscr{N}$.

For any $i=\left(i_{1}, i_{2}, \ldots\right) \in \mathscr{N}$ and any integer $n \geqslant 1$, we denote

$$
i \mid n=\left(i_{1}, \ldots, i_{n}\right) \text {. }
$$

The same symbol will be used to denote finite sequences of integers. The set

$$
\mathscr{N}_{i \mid n}=\{j \in \mathcal{N}|i| n=j \mid n\}
$$

is called Baire interval of rank $n$. Baire intervals are both open and closed in $\mathscr{N}$ and

$$
\operatorname{diam} \mathscr{N}_{i \mid n} \leqslant 2^{-(n-1)} \text {. }
$$

Let $\mathscr{N}_{1}^{k}, \mathscr{N}_{2}^{k}, \ldots$ be an ordering of Baire intervals of rank $k$. In what follows, we shall fix such an ordering for every $k$. We shall consider also the set of all pairs of positive integers together with the lexicographical ordering: $(l, s)<(k, q)$ if either $l<k$ or $l=k$ and $s<q$. The lexicographical ordering is complete, i.e. any two pairs are comparable and any collection of pairs has a unique lexicographically minimal element.

Let $A \subset \mathscr{N}$. Consider the set

$$
L(A)=\left\{(k, q) \mid A \cap \mathcal{N}_{q}^{k} \neq \varnothing, A \cap C \mathscr{N}_{q}^{k} \neq \varnothing\right\}
$$

(where $C B$ is the complement of $B$ ), and let $(k(A), q(A))$ be the (lexicographically) minimal element of $L(A)$. We set

$$
\begin{gathered}
\mathscr{T} A=\left\{\begin{array}{lll}
A \cap \mathcal{N}_{q(A)}^{k(A)}, & \text { if } & L(A) \neq \varnothing, \\
\varnothing, & \text { if } & L(A)=\varnothing ;
\end{array}\right. \\
\mathscr{T}_{c} A=\left\{\begin{array}{lll}
A \cap C \mathcal{N}_{q(A),}^{k(A)}, & \text { if } & L(A) \neq \varnothing, \\
\varnothing, & \text { if } & L(A)=\varnothing .
\end{array}\right.
\end{gathered}
$$

Thus the operations $\mathscr{T}$ and $\mathscr{T}_{c}$ divide $A$ in two parts. We shall make use of certain properties of the operations which are listed below.

I. $L(A) \neq \varnothing$ iff $A$ contains more than one element.

II. $\left(\mathscr{T} A \cap \mathscr{T}_{c} A\right)=\varnothing$; if $L(A) \neq \varnothing$, then $(\mathscr{T} A) \cup\left(\mathscr{T}_{c} A\right)=A$.

III. If $A$ is closed (resp. open, perfect), then so are $\mathscr{T}_{A}$ and $\mathscr{T}_{c} A$.

Indeed, Baire intervals are closed, open and contain no isolated points.

Let us denote $\mathscr{T}(\mathscr{T} A)=\mathscr{T}^{2} A$ etc.

IV. Let $B=\mathscr{T}^{m_{k}} \mathscr{T}_{c}^{s_{k}} \ldots \mathscr{T}^{m_{1}} \mathscr{T}_{c}^{s_{1}} A$. If $B \neq \varnothing$ and $m_{1}+\ldots+m_{k}>0$, then

$$
\operatorname{diam} B \leqslant 2^{-\left(m_{1}+\ldots+m_{k}-1\right)} .
$$

Indeed, take any $C$. If $C$ belongs to a Baire interval of rank $n$, then $k(C) \geqslant n+1$. Hence $\mathscr{T} C$ belongs to a Baire interval of rank $n+1$. This is also true if we consider $\mathscr{N}$ as the unique Baire interval of rank 0. It follows that $B$ belongs to a Baire interval of rank $m_{1}+\ldots+m_{k}$ which implies the desired inequality.

V. $\bigcap_{s=1}^{\infty} \mathscr{T}_{c}^{s} A$ contains at most one element.

Assume the contrary: the intersection contains two different elements $i$ and $j$. Let $k_{s}=k\left(\mathscr{T}_{c}^{s-1} A\right), q_{s}=q\left(\mathscr{T}_{c}^{s-1} A\right)$. By definition, $\left(k_{s}, q_{s}\right)$ is the minimal element of $L\left(\mathscr{T}_{c}^{s-1} A\right)$ and $\left(k_{s}, q_{s}\right) \notin L\left(\mathscr{T}_{c}^{s} A\right)$. Since $\mathscr{T}_{c}^{s} A \subset \mathscr{T}_{c}^{s-1} A$ and hence $L\left(\mathscr{T}_{c}^{s} A\right)$. $c L\left(\mathscr{T}_{c}^{s-1} A\right)$, it follows that

$$
\left(k_{1}, q_{1}\right) \prec\left(k_{2}, q_{2}\right) \prec \ldots
$$

Since $i \neq j$, there is a pair $(k, q)$ such that, say, $i \in \mathscr{N}_{q}^{k}$ and $j \notin \mathscr{N}_{q}^{k}$. Both $i$ and $j$ belong to any $\mathscr{T}_{c}^{s} A$, hence $(k, q) \in L\left(\mathscr{T}_{c}^{s} A\right)$ for any $s=1,2, \ldots$ Therefore $\left(k_{s}, q_{s}\right) \prec(k, q)$ for all $s$. It is easy to see that this contradicts to (1). 
Let $R$ be a multifunction from $T$ into $\mathscr{N}$. Applying $\mathscr{T}$ - and $\mathscr{T}_{c^{-}}$operations to every $R(t)$, we get a pair of new multifunctions $\mathscr{T} R$ and $\mathscr{T}_{c} R$. We write

$$
R^{-}(A)=\{t \in T \mid R(t) \cap A \neq \varnothing\} .
$$

VI. Let $\mathfrak{M}_{\mathscr{U}}(R)$ be the $\sigma$-algebra generated by the sets $R^{-}(U), U \subset \mathscr{N}$ being open. Then

$$
(\mathscr{T} R)^{-}(U) \in \mathfrak{M}_{\mathscr{U}}(R), \quad\left(\mathscr{T}_{c} R\right)^{-}(U) \in \mathfrak{M}_{\mathfrak{Z}}(R)
$$

whenever $U \subset \mathcal{N}$ is open

Indeed, let

$$
\begin{aligned}
& G_{q}^{k}(R)=R^{-}\left(\mathscr{N}_{q}^{k}\right) \cap R^{-}\left(C \cdot N_{q}^{k}\right), \\
& T_{q}^{k}(R)=\bigcup_{(l, s)<(k, q)} G_{s}^{l}(R) .
\end{aligned}
$$

Then $(\mathscr{T} R)(t)=R(t) \cap \mathscr{N}_{q}^{k}$ iff $t \in G_{q}^{k}(R) \backslash T_{q}^{k}(R)$. We have therefore

$$
(\mathscr{T} R)^{-}(U)=\bigcup_{k, q}\left(\mathrm{R}^{-}\left(U \cap \mathcal{N}_{q}^{k}\right) \cap\left(G_{q}^{k}(R) \backslash T_{q}^{k}(R)\right)\right) .
$$

Replacing $\mathscr{N}_{q}^{k}$ by their complements, we get the formula for $\left(\mathscr{T}_{c} R\right)^{-}(U)$. It remains to note that $G_{q}^{k}(R), T_{q}^{k}(R), R^{-}\left(U \cap \mathscr{N}_{q}^{k}\right)$ and $R^{-}\left(U \cap C \mathscr{N}_{q}^{k}\right)$ belongs to $\mathfrak{M}_{\mathfrak{l l}}(R)$ since Baire intervals and their complements are open.

VII. Let $\mathscr{T}_{c_{\Delta}}^{\infty}(R)=\left\{(t, i) \mid i \in \bigcap_{s} \mathscr{T}_{c}^{s} R(t)\right\}$. If $R$ is closed-valued, then

$$
\mathscr{T}_{c}^{\infty}(R) \in \mathfrak{M}_{\mathscr{U}}(R) \otimes \mathfrak{B}(\mathscr{N}) .
$$

Indeed, since $R$ is closed-valued, so are all $\mathscr{T}_{c}^{s} R$ (according to III). By VI these multifunctions are also $\mathfrak{M}_{\mathscr{U}}(R)$-measurable, hence $\operatorname{Gr} \mathscr{T}_{c}^{s} R=\left\{(t, i) \mid i \in \mathscr{T}_{c}^{s} R(t)\right\}$ belongs to $\mathfrak{M}_{\mathscr{q}}(R) \otimes \mathfrak{B}(\mathscr{N})$ for all $s$. But

$$
\mathscr{T}_{c}^{\infty}(R)=\bigcap_{s} \operatorname{Gr} \mathscr{T}_{c}^{s} R
$$

\$3. Proof of the theorem. We shall consider four successively extending situations, the final situation coinciding with the general setting of the statement. To begin with, recall the following well-known fact: if $(T, \mathfrak{M}, \mu)$ is a space with a complete positive $\sigma$-finite measure and $R$ is a closed-valued multifunction from $T$ into a Polish space $X$, then the properties

\section{Gr $R \in \mathfrak{M} \otimes \mathfrak{B}(X)$ and}

$R$ is $\mathfrak{M}$-measurable (i.e. $R^{-}(U) \in \mathfrak{M}$ for any open $U \subset X$ )

are equivalent.

Step 1. The theorem is true under the additional assumption that $X=\mathscr{N}$ and $M(t)$ is a perfect set for any $t \in T$.
Let

$$
\begin{aligned}
M_{i \mid 0} & =M, \quad \forall i \in \mathscr{N}, \\
M_{i \mid n}(t) & =\mathscr{T T}_{c}^{i_{n}-1} \ldots \mathscr{T} \mathscr{T}_{c}^{i_{1}-1} M(t), \forall t \in T, \forall i \in \mathscr{N}, \\
T_{i \mid n} & =\operatorname{pr}_{T} \mathscr{T}_{c}^{\infty}\left(M_{i \mid n}\right)
\end{aligned}
$$

$\left(\operatorname{pr}_{T}\right.$ denotes projection on $\left.T\right)$, and let $x_{i \mid n}(t)$ be the unique element of $\bigcap_{s} \mathscr{T}_{c}^{s} M_{i] n}(t)$ for $t \in T_{i \mid n}$ (see V).

(a) $M_{i \mid n}(t)$ is a nonempty perfect set for all $i \in \mathcal{N}, n \geqslant 0, t \in T$.

This follows from III.

(b) $M_{i \mid n}(U) \in \mathfrak{M}_{\mathscr{q}}(M) \subset \mathfrak{M}$ whenever $U \subset \mathcal{N}$ is open.

The left inclusion follows from VI and the second from the above mentioned equivalence (since perfect sets are closed).

(c) $T_{i \mid n} \in \mathfrak{M}$ and $x_{i \mid n}($.$) is \mathfrak{M}$-measurable.

This is a consequence of VII.

(d) $M_{i \mid(n-1)}(t)=\left(\bigcup_{j \in \mathscr{N}_{i \mid(n-1)}} M_{j \mid n}(t)\right) \cup\left\{x_{i \mid(n-1)}(t)\right\}$.

Here we have set $\left\{x_{i \mid n}(t)\right\}=\varnothing$ if $t \notin T_{i \mid n}$. Note also that though Baire intervals are uncountable, any set $\{j|n| j|(n-1)=i|(n-1)\}$ is countable so that the union in the formula contains countably many different members.

The very equality follows directly from II, V and definitions.

(e) If $i|n \neq j| n$, then $M_{i \mid n}(t) \cap M_{j \mid n}(t)=\varnothing$ and $x_{i \mid n}(t) \neq x_{j \mid n}(t)$ for any $t$ $x_{i \mid(n-1)}(t) \notin M_{j \mid n}(t)$ for any $i, j, n, t$.

(f) $\operatorname{diam} M_{i \mid n}(t) \leqslant 2^{-(n-1)}$.

This follows from IV.

(g) For any $t \in T$ the formula

$$
h(t, i)=\bigcap_{n} M_{i \mid n}(t)
$$

defines a one-to-one mapping from $\mathscr{N}$ into $\mathscr{N}$.

Indeed, the intersection contains exactly one element for any $t \in T ; i \in \mathscr{N}$ which follows from (a) and (f). On the other hand, $h(t, i) \neq h(t, j)$ if $i \neq j$ by virtue of (e).

(h) The function $i \rightarrow h(t, i)$ is continuous for any $t \in T$.

Indeed, let $b\left(j_{1}, j_{2}\right) \leqslant 2^{-(n+2)}$. This means that $j_{1}$ and $j_{2}$ belong to the same Baire interval of rank $n$, say to $\mathscr{N}_{i \mid n}$; hence $j_{1}\left|n=j_{2}\right| n=i \mid n$ and by definition, $h\left(t, j_{1}\right)$ $\in M_{i \mid n}(t), h\left(t, j_{2}\right) \in M_{i \mid n}(t)$. Applying (f), we get $b\left(h\left(t, j_{1}\right), h\left(t, j_{2}\right)\right) \leqslant 2^{-(n-1)}$.

(i) The function $t \rightarrow h(t, i)$ is $\mathfrak{M}$-measurable for any $i \in \mathscr{N}$. 
Indeed, since $M_{i \mid n}(t)$ are closed,

$$
\{t \mid h(t, i) \in F\}=\bigcap_{\substack{\varepsilon>0 \\ n \geqslant 1}} M_{i \mid n}^{-}\left(F_{\varepsilon}\right)
$$

whenever $F \subset \mathscr{N}$ is closed. Here $F_{\varepsilon}=\{j \mid b(j, F)<\varepsilon\}$.

Let $\varphi$ be a one-to-one mapping from $N$ onto the set of all finite sequences of integers. For simplicity, we shall write $x_{k}($.$) and T_{k}$ instead $x_{\varphi(k)}($.$) and T_{\varphi(k)}$. Let $N(t)=\left\{k \in N \mid t \in T_{k}\right\}$.

(j) For any $t \in T$,

$$
h(t, \mathscr{N})=M(t) \backslash\left\{x \in \mathscr{N} \mid x=x_{k}(t) \text { for some } k \in N(t)\right\} .
$$

Indeed, the inclusion $h(t, \mathscr{N}) \subset M(t)$ is obvious from (d) and $(\mathrm{g})$. On the other hand, again according to (d) and (g), if $x \in M(t)$ then either $x=x_{i \mid n}(t)$ for some $i \mid n$ or there is a sequence $\left\{i^{k}\right\}$ of elements of $\mathscr{N}$ such that $i^{k+1}\left|k=i^{k}\right| k$ for all $k$ and $x \in M_{i^{k} \mid k}(t)$ for all $k$. The first relation shows that $\left\{i^{k}\right\}$ converges to certain $i$ when $k \rightarrow \infty$ and $i^{k}|k=i| k$ for all $k$. Hence $x \in M_{i \mid n}(t)$ for all $n$ which means by definition that $x=h(t, i)$.

Let us choose a countable family of different elements of $\mathscr{N}$ which we shall write in the form $i_{k r}, k, r=1,2, \ldots$, and consider the functions

$$
y_{k r}(t)= \begin{cases}h\left(t, i_{k r}\right), & \text { if } t \notin T_{k}, \\ x_{k}(t), & \text { if } t \in T_{k}, r=1, \\ h\left(t, i_{k(r-1)}\right), & \text { if } t \in T_{k}, r>1 .\end{cases}
$$

The set $\mathcal{N} \backslash\left\{i_{k r}\right\}$ is a $G_{\delta}$. Therefore it is homeomorphic to a Polish space $Z^{\prime}$. Let $\psi: Z^{\prime} \rightarrow \mathscr{N}$ be the corresponding homeomorphism. Consider the space $Z=Z^{\prime} \cup(N \times N)$ (also Polish if $N$ is supplied with the discrete topology) and define $f: T \times Z \rightarrow \mathscr{N}$ as follows:

$$
f(t, z)=\left\{\begin{array}{lll}
h(t, \psi(z)), & \text { if } & z \in Z^{\prime} \\
y_{k r}(t), & \text { if } & z=(k, r) \in N \times N .
\end{array}\right.
$$

Then $f(., z)$ is measurable (by (c) and (i)), $f(t,$.$) is continuous (by (h)) and one-to-one$ (by (e), (g) and since $i_{k r}$ are different). Finally, $f(t, Z)=M(t)$ which follows from $(\mathrm{j})$ and the definition of $y_{k r}($.$) .$

Step 2. The theorem is true under the additional assumption that $X=\mathscr{N}$ and $M(t)$ is closed for any $t \in T$.

To prove the theorem in this case, we consider a transfinite sequence of triples. $\left(M_{\alpha}, T_{\alpha}, x_{\alpha}().\right)(\alpha<\Omega, \Omega$ being the first uncountable ordinal) where for any ordinal $\alpha$ $M_{\alpha}$ is a closed-valued $\mathfrak{M}$-measurable multifunction from $T$ into $\mathscr{N}, T_{\alpha} \subset T$ and $x_{\alpha}():. T_{\alpha} \rightarrow \mathscr{N}$. The triples will be defined inductively as follows:

$$
M_{1}=M
$$

If for a given ordinal $\alpha, M_{\alpha}$ is already defined, we set

$$
\begin{gathered}
T_{\alpha}=\left\{t \in T \mid M_{\alpha}(t) \text { has an isolated point }\right\}, \\
x_{\alpha}(t)=x_{\alpha k}(t), \quad \text { if } \quad t \subseteq T_{\alpha k} \backslash \bigcup_{s<k} T_{\alpha s},
\end{gathered}
$$

where $\left\{x_{\alpha 1}(),. x_{\alpha 2}(),. \ldots\right\}$ is an arbitrarily chosen Castaing representation for $M_{\alpha .}$ (see [5]) and for any fixed $k$

$$
T_{\alpha k}=\left\{t \in T \mid \inf _{s \neq k} b\left(x_{\alpha k}(t), x_{\alpha s}(t)\right)>0\right\}
$$

and

$$
\left[M_{\alpha+1}(t)=\left\{\begin{array}{lll}
M_{\alpha}(t), & \text { if } & t \notin T_{\alpha}, \\
M_{\alpha}(t) \backslash\left\{x_{\alpha}(t)\right\}, & \text { if } & t \in T_{\alpha}
\end{array} ;\right.\right.
$$

If the set of ordinal which are less then $\alpha$ does not contain maximal element and $M_{\xi}$ is defined for any $\xi<\alpha$, we set

$$
M_{\alpha}=\bigcap_{\xi<\alpha} M_{\xi} .
$$

First we shall verify that this definition is correct.

(k) For any countable ordinal $\alpha, M_{\alpha}$ is an $\mathfrak{M}$-measurable multifinction with uncountable and closed values, $T_{\alpha} \in \mathfrak{M}$ and $x_{\alpha}($.$) is \mathfrak{M}$-measurable.

Indeed, if $M_{\alpha}$ is defined by (3) it is closed-valued and $\mathfrak{M}$-measurable if so are $M_{\xi}$, $\xi<\alpha$; the first is obvious and the second follows from the fact that the graph of $M_{\alpha-}$ is the intersection of countably many subsets of $\mathfrak{M} \otimes \mathfrak{B}(\mathcal{N})$. Let $M_{\alpha+1}$ be defined by (2). First we note that whenever $x$ is an isolated point of $M_{\alpha}(t)$ and

$$
\left\{x_{\alpha 1}(.), x_{\alpha 2}(.), \ldots\right\}
$$

is a Castaing representation for $M_{\alpha}$, there is $k$ such that $x_{\alpha k}(t)=x$. It follows that $T_{\alpha}=\bigcup T_{\alpha k} \in \mathfrak{M}, x_{\alpha}($.$) is \mathfrak{M}$-measurable and for any $t \in T_{\alpha}, x_{\alpha}(t)$ is an isolated point of $M_{\alpha}(t)$ which in turn shows that $M_{\alpha+1}$ is closed-valued and the graph of $M_{\alpha+1}$ belongs to $\mathfrak{M} \otimes \mathfrak{B}(\mathscr{N})$, hence $M_{\alpha+1}$ is $\mathfrak{M}$-measurable (thanks to the equivalence mentioned in the beginning of the proof).

Finally, since $M(t)$ are uncountable and any difference $M_{\alpha}(t) \backslash M_{\alpha+1}(t)$ contains. at most one point, every $M_{\alpha}(t)$ is also uncountable.

(l) If $M_{\alpha}(t)=M_{\alpha+1}(t)$ then $M_{\alpha}(t)=M_{\beta}(t)$ for all $\beta>\alpha$.

Indeed, in this case, $M_{\alpha}(t)$ is a perfect set.

(m) $x_{\alpha}(t) \neq x_{\beta}(t)$ if $\alpha \neq \beta$.

(n) There are $T^{\prime} \subset T$ with $\mu\left(T \backslash T^{\prime}\right)=0$ and a countable ordinal $\alpha$ such that $M_{\alpha}(t)=M_{\beta}(t)$ for all $\beta>\alpha, t \in T^{\prime}$.

Since $T$ is a countable union of sets with finite measure no loss of generality wilt: follow if we assume that $\mu T<\infty$. 
Let $\left\{x_{1}, x_{2}, \ldots\right\}$ be a dense countable subset in $\mathscr{N}$. To any closed set $A \subset \mathscr{N}$ we can assign the quantity

$$
r(A)=\sum_{k=1}^{\infty} 2^{-k} b\left(x_{k}, A\right) .
$$

Since $b(i, j) \leqslant 1, r(A) \leqslant 1$ for any $A$. If $R$ is a closed-valued multifunction from $T$ into $\mathscr{N}$ then the real-valued function $t \rightarrow r(R(t))$ is $\mathfrak{M}$-measurable and even summable since $\mu(T)<\infty$ by the assumption. Let

$$
r_{\alpha}=\int_{T} r\left(M_{\alpha}(t)\right) d \mu .
$$

Then $r_{1} \leqslant r_{2} \leqslant \ldots \leqslant r_{\alpha} \leqslant \ldots$ (because $M_{1} \supset M_{2} \supset \ldots$ ). Moreover $r_{\alpha}<r_{\alpha+1}$ iff $M_{\alpha}(t)$ $\neq M_{\alpha+1}(t)$ on a set of positive measure because $A \subset B, A \neq B$ (both $A$ and $B$ are closed) implies $r(A)>r(B)$.

Thus only two cases are concievable. Either there is an $\alpha<\Omega$ such that $r_{\alpha}=r_{\alpha+1}$ or $r_{\alpha}<r_{\alpha+1}$ for all $\alpha<\Omega$. The second case is impossible since only countably many strictly increasing real numbers can exist. In the first case which is threreby the only possible case $M_{\alpha}(t)=M_{\alpha+1}(t)$ on a set $T^{\prime}$ with $\mu T^{\prime}=\mu T$ which in view of (l) yields the desired result.

Let $M_{0}(t)$ be the perfect kernel of $M(t)$.

(o) $M_{0}$ is a closed-valued $\mathfrak{M}$-measurable multifunction and there is a sequence $\left\{x_{k}().\right\}$ of $\mathfrak{M}$-measurable mappings into $\mathscr{N}$, each defined on a set $T_{k} \in \mathfrak{M}$, such that $x_{k}(t) \neq x_{s}(t)$ for any $t$ if $k \neq s, x_{k}(t) \notin M_{0}(t)$ and

$$
M(t)=M_{0}(t) \cup\left\{x \in \mathcal{N} \mid x=x_{k}(t) \text { for some } k \in N(t)\right\},
$$

where, as before, $N(t)=\left\{k \in N \mid t \in T_{k}\right\}$.

Let $\alpha$ and $T^{\prime}$ be the same as in (n). Then $M_{0}(t)=M_{\alpha}(t)$ for all $t \in T^{\prime}$ and hence, as far as $\mu$ has been assumed complete, $M_{0}$ is an $\mathfrak{M}$-measurable multifunction.

Denote $T^{\prime \prime}=T \backslash T^{\prime}$. For any $t \in T^{\prime \prime}$, the set $M(t) \backslash M_{0}(t)$ is at most countable. Numbering arbitrarily elements of any such difference (which can be done only with the help of Zermelo choice axiom), we shall find a decreasing sequence $T_{1}^{\prime \prime} \supset T_{2}^{\prime \prime} \supset \ldots$ of subsets of $T^{\prime \prime}$ and a sequence $\left\{x_{k}^{\prime \prime}().\right\}$ of mappings respectively from $T_{k}^{\prime \prime}$ into $\mathscr{N}$ such that for any $t \in T^{\prime \prime}$

$$
M(t)=M_{0}(t) \cup\left\{x_{k}^{\prime \prime}(t) \mid k \in N^{\prime \prime}(t)\right\}
$$

$\left(N^{\prime \prime}(t)=\left\{k \in N \mid t \in T_{k}^{\prime \prime}\right\}\right)$. Since $\mu T^{\prime \prime}=0$ and $\mu$ is complete, every $T_{k}^{\prime \prime}$ and every $x_{k}^{\prime \prime}($.$) is \mathfrak{M}$-measurable;

Let now $\varphi$ be a one-to-one mapping from $N$ onto the set of ordinals which are $\leqslant \alpha$. We shall write $x_{k}^{\prime}()=.x_{\varphi(k)}(),. T_{k}^{\prime}=T_{\varphi(k)} \backslash T^{\prime \prime}$. According to definition, for $t \in T^{\prime}$

$$
M(t)=M_{0}(t) \cup\left\{x_{k}^{\prime}(t) \mid k \in N(t)\right\} .
$$

It remains to set

$$
\begin{gathered}
T_{k}=T_{k}^{\prime} \cup T_{k}^{\prime \prime}, \\
x_{k}(t)= \begin{cases}x_{k}^{\prime \prime}(t), & \text { if } t \in T_{k}^{\prime \prime}, \\
x_{k}^{\prime}(t), & \text { if } t \in T_{k}^{\prime} .\end{cases}
\end{gathered}
$$

According to what was proved at Step $1, M_{0}(t)=h(t, Y)$ where $Y$ is a Polish space and $h(t, y)$ is $\mathfrak{M}$-measurable in $t$ and continuous and one-to-one in $y$.

The situation we have now is just the same as the one we had at the end of Step 1. Having done the same as there, we shall find the desired Polish space $Z$ and mapping $f(t, z): T \times Z \rightarrow \mathscr{N}$.

Step 3. The theorem is true under the additional assumption that $M$ is closedvalued.

As well known, any Polish space is one-to-one continuous image of a closed subset of $\mathscr{N}$. Let $Q \subset \mathscr{N}$ be closed and $\psi: Q \rightarrow X$ be one-to-one continuous and such that $\psi(Q)=X$. We set

$$
R(t)=\psi^{-1}(M(t))
$$

Then $R(t)$ is an uncountable closed subset of $\mathscr{N}$ for any $t \in T$. If $B \subset \mathscr{N}$ is Borel, then so are $B \cap Q$ and $\psi(B)=\psi(B \cap Q)$. Hence

$$
R^{-}(B)=(\psi(B \cap Q)) \in \mathfrak{M},
$$

since $\mu$ is a complete measure. This shows that $R$ is $\mathfrak{M}$-measurable and hence the graph of $R$ belongs to $\mathfrak{M} \otimes \mathfrak{B}(\mathscr{N})$. According to Step 2, there are a Polish space $Z$ and a Carathéodory mapping $h: T \times Z \rightarrow \mathscr{N}$ which is one-to-one in $z$ and such that $h(t, Z)=R(t)$ for all $t$. It remains to set $f(t, z)=\psi(h(t, z))$.

Step 4. The theorem is true.

To prove this, it suffices to show that

(p) There are a Polish space $Y$, a continuous mapping $\psi: Y \rightarrow X$, and an Mi-measurable closed-valued multifunction $R$ from $T$ into $Y$ such that

$$
M(t)=\psi(R(t)), \quad \forall t \in T
$$

and $\psi$ is one-to-one on every $R(t)$.

The proof of this fact is more or less traditional; it repeats essentially the proof of the well-known theorem that any Borel set in a Polish space is a one-to-one continuous image of a null-dimensional Polish space (cf. [6], § 37, I). Therefore we shall omit certain routine details in the proof to follow.

(q) The family $\mathfrak{M} \otimes \mathfrak{B}(X)$ is the minimal family which contains

all sets $G \times B, G \in \mathfrak{M}, B \subset \mathfrak{B}(X)$;

countable intersections of its elements;

countable unions of disjoint elements. 
The proof is based on simple set-theoretic arguments repeating almost wordto-word those which can be found in ([3], § 30, V).

Any subset of $T \times X$ is obviously the graph of a set-valued mapping from $T$ into $X$. We shall call the set $A \in \mathfrak{M} \otimes \mathfrak{B}(X)$ well-representable if there are a Polish space $Z$, a closed-valued $\mathfrak{M}$-measurable multifunction $R$ from $E=\operatorname{pr}_{T} A$ into $Z$ and a continuous mapping $g: Z \rightarrow X$ which is one-to-one on every $R(t)$ and such that

$$
A=\{(t, g(z)) \mid t \in E, z \in R(t)\} .
$$

To prove (p), it suffices to show that any element of $\mathfrak{M} \otimes \mathfrak{B}(X)$ is well-representable.

(r) A rectangle $G \times B$ is well-representable whenever $G \in \mathfrak{M}, B \in \mathfrak{B}(X)$.

Indeed, take a Polish space $Z$ and a one-to-one continuous mapping $g: Z \rightarrow X$ such that $g(Z)=B$ and set $R(t)=Z$ for $t \in G$.

(s) A countable intersection of well-representable sets is also well-representable.

Let $A_{1}, A_{2}, \ldots$ be well-representable, and let $Z_{1}, Z_{1}, \ldots, R_{1}, R_{2}, \ldots, g_{1}, g_{2}, \ldots$ be the corresponding Polish spaces, closed-valued $\mathfrak{M}$-measurable multifunctions and mappings. Since $A_{k}$ belong to $\mathfrak{M} \otimes \mathfrak{B}(X)$, so does $A=\bigcap A_{k}$.

Consider the product space $Z^{\infty}=Z_{1} \times Z_{2} \times \ldots$ (which is also Polish) and the set-valued mapping $R^{\infty}(t)=R_{1}(t) \times R_{2}(t) \ldots$ from $T$ into $Z$. Ir is easy to verify that $\operatorname{Gr} R^{\infty} \in \mathfrak{M} \otimes \mathfrak{B}(Z)$ (since $\operatorname{Gr} R_{k} \in \mathfrak{M} \otimes \mathfrak{B}\left(Z_{k}\right)$ ). Consider also for any $t \in E$ $=\operatorname{pr}_{T} A$ the set

$$
Q=\left\{z=\left(z_{1}, z_{2}, \ldots\right) \in Z^{\infty} \mid g_{1}\left(z_{1}\right)=g_{2}\left(z_{2}\right)=\ldots\right\} .
$$

This set is obviously closed. It is easy to see that $(t, x) \in A$ iff there is $z \in R^{\infty}(t) \cap Q$ such that $g_{k}\left(z_{k}\right)=x$ for all $k$.

The set-valued mapping $t \rightarrow R(t)=R^{\infty}(t) \cap Q$ is closed-valued and $\operatorname{Gr} R$ $\in \mathfrak{M} \otimes \mathfrak{B}\left(Z^{\infty}\right)$, hence it is $\mathfrak{M}$-measurable. On the other hand, the mapping

$$
z=\left(z_{1}, z_{2}, \ldots\right) \rightarrow g(z)=g_{1}\left(z_{1}\right)
$$

is continuous and one-to-one on $Q$ and hence on every $R(t)$. It remains to observe that (4) obviously holds for our $A$ and $R(t)$.

( $\mathrm{t})$ A countable union of disjoint well-representable sets is also well-representable.

Let $A_{1}, A_{2}, \ldots$ be well-representable, $A_{k} \cap A_{1}=\varnothing, k, l=1,2, \ldots$, and let $Z_{1}, Z_{2}, \ldots, R_{1}, R_{2}, \ldots, g_{1}, g_{2}, \ldots$ be corresponding Polish spaces, closed-valued $\mathfrak{M}$-measurable multifunctions and mappings. Let $Z$ be the topological direct sum of $Z_{k}$, that is $Z=Z_{1} \cup Z_{2} \cup \ldots\left(Z_{k}\right.$ are considered as disjoint sets) endowed with the strongest topology in which the imbeddings $Z_{k} \rightarrow Z$ are continuous, and let $R(t)$ $=R_{1}(t) \cup R_{2}(t) \ldots$ Again no difficulties is connected with verifying that $R$ is closedvalued and $\mathrm{Gr} R \in \mathfrak{M} \otimes \mathfrak{B}(Z)$. The mapping $z \rightarrow g(z)=g_{k}(z)$ if $z \in Z_{k}$ from $Z$ into $X$ is continuous and one-to-one on every $R(t)$ (since $g_{k}$ are one-to-one on $R_{k}(t)$ and $g_{k}\left(R_{k}(t)\right)$ do not meet each other). If $A=\bigcup A_{k}$, then (4) holds and hence $A$ is well-representable.

As follows immediately from (q)-(t), any element of $\mathfrak{M} \otimes \mathfrak{B}(X)$ is well-representable. This proves $(p)$ and hence the theorem.

\section{References}

[1] D. Cenzer and R. D. Mauldin, Measurable parametrizations and selections, Trans. Amer. Math. Soc. 245 (1978), pp. 399-408.

[2] A. D. Ioffe, Single-valued representations of set-valued mappings, Trans. Amer. Math. Soc. 252 (1979), pp. 133-145.

[3] K. Kuratowski, Topology, Vol. 1, New York-London-Warszawa 1966.

[4] N. Lusin, Leçons sur les Ensembles Analytiques et Leurs Applications, Gauthier-Villars 1930.

[5] D. H. Wagner, Survey on measurable selection theorems, SIAM J. Control and Optimization 15 (1977), pp. 859-903.

[6] E. Wesley, Extensions of measurable choice by means of forsing, Israel J. Math. 14 (1973), pp. 104-111. 